\title{
The Molecular Gas Component of Galaxy Disks
}

\author{
Leo Blitz \\ University of California, Berkeley \\ Radio Astronomy Lab., 601 Campbell Hall \\ Berkeley, CA 94720-3411, USA \\ email: blitz@astro.berkeley.edu
}

\begin{abstract}
The molecular gas in galaxy disks shows much more galaxy to galaxy variation than does the atomic gas. Detailed studies show that this variation can be attributed to differences in hydrostatic pressure in the disks due largely to variations in the stellar surface density and the total gas surface density. One prediction of pressure modulated $\mathrm{H}_{2}$ formation is that the location where $\mathrm{HI}$ and $\mathrm{H}_{2}$ have equal surface densities occurs at a constant value of the stellar surface density in the disk. Observations confirm this constancy to $40 \%$.
\end{abstract}

Keywords. star formation, galaxies, galaxy disks

\section{Introduction}

Among the relatively few statements that can be made with confidence about star formation in galaxies, one stands out: stars form from clouds of molecular gas, now and always. We know this empirically at the present and earlier epochs from observations that show that the youngest stars are always found embedded in molecular gas. From theory, astronomers generally believe that stars form as the result of a Jeans instability. In the form of the Jeans density, we can write:

$$
\rho_{\text {Jeans }}=\left(\frac{k T}{\mu m_{H} G}\right)^{3} \frac{\pi^{5}}{\left(6 M_{J}\right)^{2}}
$$

Since $\mathrm{T}$ is typically $\sim 10 \mathrm{~K}$ and can, in any event, never be less than $2.7 \mathrm{~K}$, to form a one solar mass star requires a number density of $\sim 10^{6} \mathrm{~cm}^{-3}$, densities found only in molecular clouds. Thus, when one considers star formation in galaxies from the ISM, one need only consider the molecular gas; the atomic gas is irrelevant except as a reservoir from which molecular gas can associate.

Galaxy disks exhibit relatively little variation in HI surface density with radius and from galaxy to galaxy (for late types) except in dwarfs. Typically, the surface density peaks at about $10 \mathrm{M}_{\odot}$ and remains relatively constant throughout the stellar disk; regions with higher gas surface density are almost invariably primarily molecular. The variation in molecular gas content and surface density is however much greater than for HI. This can be seen in Figures 1 and 2. Figure 1 shows the CO emission from 20 strong CO emitters from the BIMA SONG Survey (Helfer et al. 2003). Figure 2 shows the CO emission from the remaining 24 galaxies in the survey displayed on the same scale. To be detectable at all in these images, the $\mathrm{CO}$ must have an $\mathrm{H}_{2}$ surface density of $\sim 6 \mathrm{M}_{\odot}$. The range of $\mathrm{H}_{2}$ surface densities shown in the two figures is more than two orders of magnitude. The Milky Way most closely resembles NGC 3351 in its CO content (second row and third column of Figure 2). NGC 4535 in the third row and first column of 


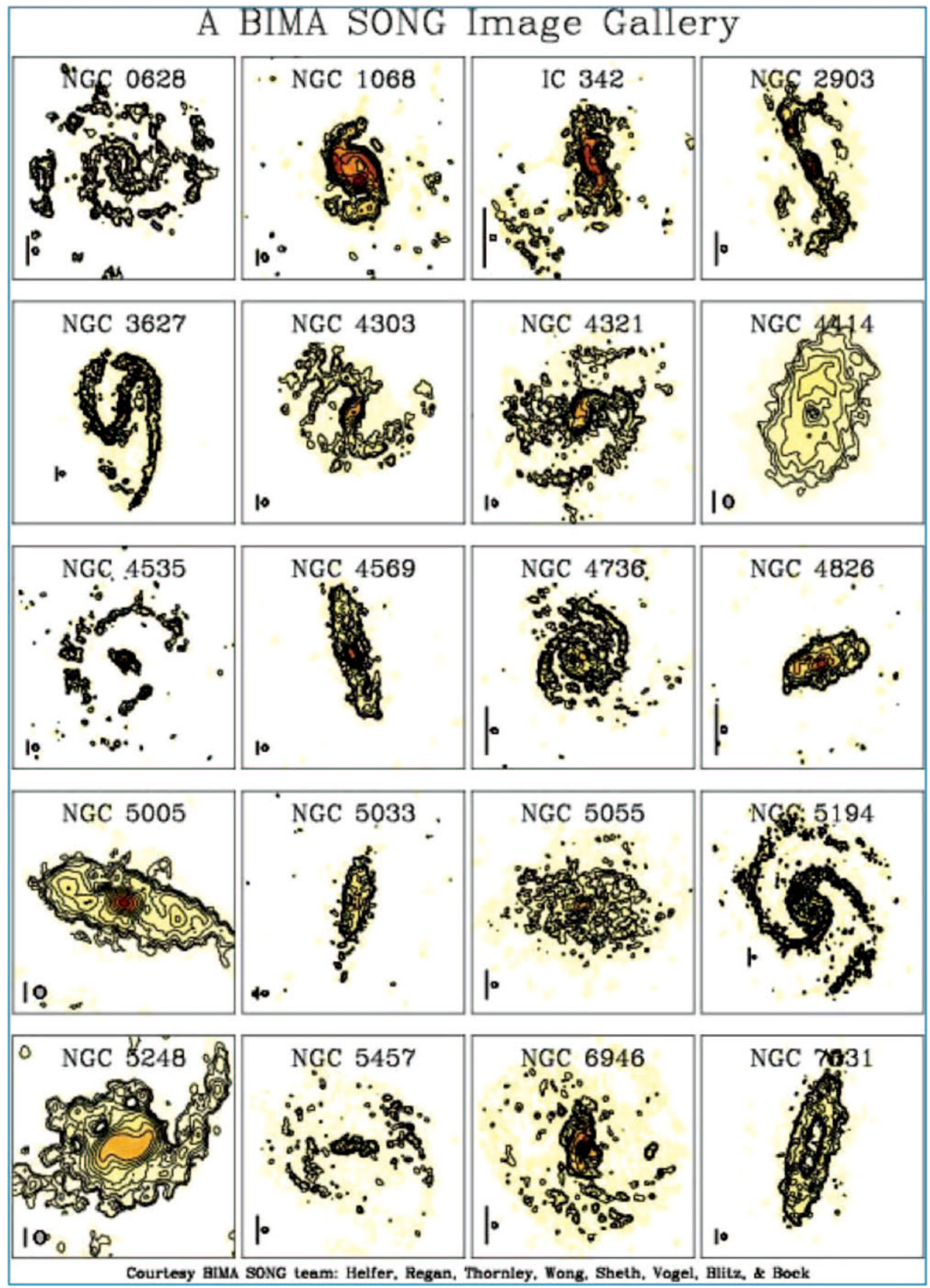

Figure 1. Maps of $\mathrm{CO}$ emission from 20 of the strongest emitters from the 44 galaxy BIMA SONG survey. Note the wide range in $\mathrm{CO}$ morphologies in the maps. REF

Figure 1 is morphologically similar, and is also a multi-armed spiral, but has a ring of emission with a higher surface density than that of the Galaxy.

What causes the differences from galaxy to galaxy? Following the suggestion of Wong and Blitz (2002), Blitz and Rosolowsky $(2004,2006)$ argued that the ratio of $\mathrm{N}\left(\mathrm{H}_{2}\right) / \mathrm{N}(\mathrm{HI})$ 

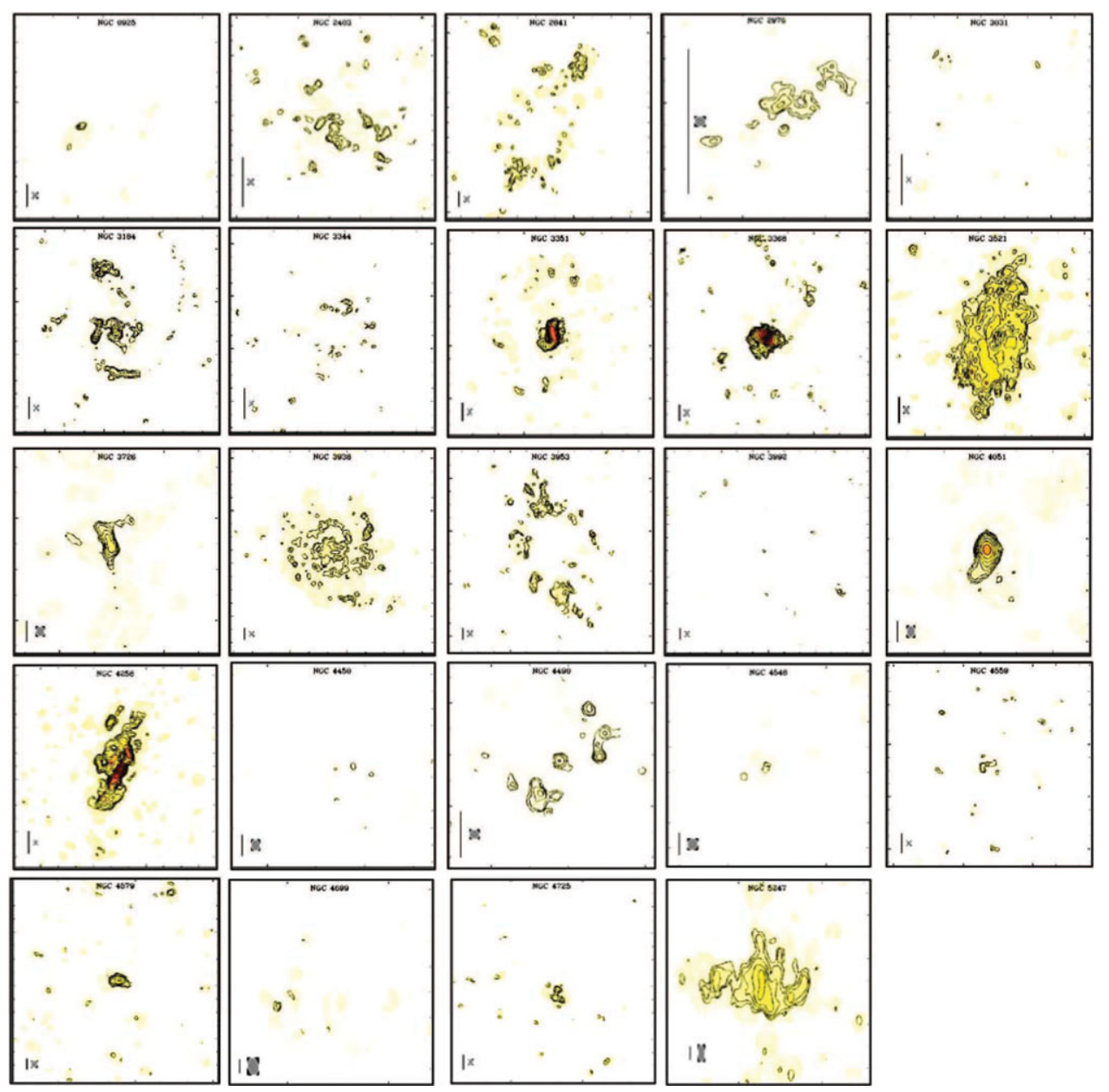

Figure 2. Maps of the $\mathrm{CO}$ emission from the remaining 24 galaxies in the survey which are not as frequently displayed as those in Figure 1. Some galaxies are not detected at all, some only in the nucleus.

on a pixel by pixel basis is primarily due to the hydrostatic pressure in the galactic disk and they empirically derived the following relation:

$$
\mathrm{R}_{m o l}=\left[\frac{P_{e x t} / k}{(3.5 \pm 0.6) \times 10^{4}}\right]^{0.92 \pm 0.07}
$$

where $\mathrm{R}_{m o l}=2 \mathrm{~N}\left(\mathrm{H}_{2}\right) / \mathrm{N}(\mathrm{HI})$.

Because galaxy disks exhibit little variation in the velocity dispersion of the gas and the scale height of the stars both within and between galaxies, one of the surprising consequences of the $\mathrm{R}_{m o l}$-pressure relation is that $\mathrm{R}_{m o l}=1$ should occur at constant stellar surface density in every galaxy. Blitz and Rosolowsky (2004) showed that for the 22 galaxies they analyzed, the constancy in stellar surface density is good to $40 \%$ even though the radius where the equivalence in hydrogen surface density varies by more than an order of magnitude (see Figure 3). From the $\mathrm{R}_{m o l}$-pressure relation, Blitz and Rosolowsky (2006) developed a star formation prescription that they argued was an improvement over the Kennicutt (1998a) star formation prescription. 


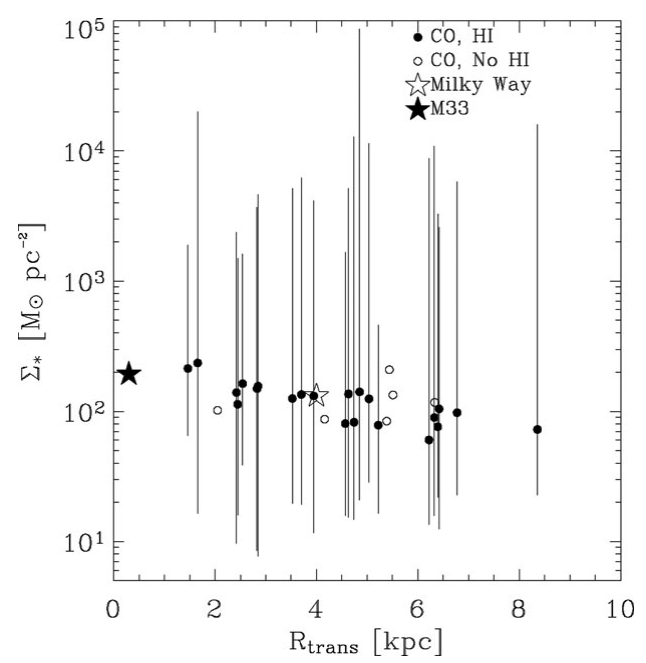

Figure 3. Plot of the median stellar surface mass density where $\mathrm{N}\left(\mathrm{H}_{2}\right) \approx N(H I)$ as a function of where this surface density occurs in the galaxy. For galaxies with measured HI densities (filled circles, 22 galaxies), the range of stellar surface densities is plotted as a vertical line (this is not an error bar), running between the values of the 1st percentile and the 99th percentile of surface density in the galaxy. Galaxies without HI measurements are plotted as open circles (6 galaxies). The stellar surface density at the transition is remarkably constant and has a mean value of $120 \mathrm{M}_{\odot} \mathrm{pc}^{-2}$ for the 22 galaxies with both $\mathrm{CO}$ and HI data. Points for the Milky Way (MW) and M33 are also plotted.

There are however sevaral other prescriptions proposed by other authors, (Wyse, 1986; Kennicutt 1998a; Elmegreen 1997; Krumholz \& McKee, 2005), some of which include cutoffs in the radial distribution of star formation effiiency in galaxies (Martin \& Kennicutt, 2001; Hunter et al. 1998; Schaye 2004; Skillman, 1987; deBlok \& Walter 2006). Martin \& Kennicutt (2001), e.g., argued that star formation cuts off beyond the radius where the disk becomes Toomre stable. Clearly, star formation prescriptions that include atomic gas must have either a cutoff in the star formation efficiency, or some sort of falloff at the edge of the stellar disk because the HI disks tend to be much more extended, often with no change in surface density at the edge of the stellar disk (Bigiel 2008).

The most extensive investigation of various star formation prescriptions and cutoffs has been done in a recent paper by Leroy et al. (2008) using the THINGS HI Survey (Walter et al. 2008), and the BIMA SONG CO Survey (Helfer et al. 2003) to get the neutral hydrogen content of a sizable number of disks. Leroy et al. (2008) used the GALEX Survey (Gil de Paz 2007) and Spitzer SINGS observations (Kennicutt et al. 2003) to measure the star formation in the disks of the same galaxies, supplemented by additional observations of their own (Leroy et al. 2009)

This vast work has come to a number of important conclusions, some of which are listed below:

1) The star formation efficiency of $\mathrm{H}_{2}$, (the rate at which a given surface density of $\mathrm{H}_{2}$ turns into stars) is constant independent of environment or any other variable they investigated. the value is $5.25 \pm 2.5 \times 10^{-10} \mathrm{yr}^{-1}$. The inverse of this quantity is 
the molecular gas depletion time, the time to use up all of the molecular gas to star formation, and is equal to $1.9 \times 10^{9} \mathrm{y}$.

2) In spiral galaxies, the transition between a mostly $\mathrm{HI}$ and a mostly $\mathrm{H}_{2}$ ISM is a well defined function of local conditions. It occurs at a characteristic radius $r=0.43 \pm$ $0.18 \mathrm{r}_{25}, \Sigma_{*}=81 \pm 25 \mathrm{M}_{\odot} \mathrm{pc}^{-2}, \Sigma_{\text {gas }}=14 \pm 6 \mathrm{M}_{\odot} \mathrm{pc}^{-2}, \mathrm{P}_{\text {hydro }}=2.3 \pm 1.5 \times 10^{4} k_{B}$ $\mathrm{cm}^{-3} \mathrm{~K}$, and $\tau_{\text {orb }}=1.8 \pm 0.4 \times 10^{8} \mathrm{yr}$.

3) $\mathrm{R}_{\text {mol }}$ appears to be a continuous function of environment from the HI dominated $\left(\mathrm{R}_{m o l} \sim 0.1\right)$ to $\mathrm{H}_{2}$ dominated $\left(\mathrm{R}_{m o l} \sim 10\right)$ regime. The variation in $\mathrm{R}_{m o l}$ is too strong to be reproduced only by varying $\tau_{\text {orb }}$ or $\tau_{f f}$. Physics other than these timescales must also play an important role in cloud formation

4) Thresholds for large scale stability do not offer an obvious way to predict $R_{m o l}$. There is no clear relationship (continuous or stepfunction) between the star formation efficiency and $\mathrm{Q}_{\text {gas }}, \mathrm{Q}_{\text {stars+gas }}$, or to a shear threshold. Disks appear to be stable or marginally stable throughout once the effects of stars are included.

5 ) The power law relationship between $R_{m o l}$ and hydrostatic pressure found by Leroy et al. (2008) is roughly consistent with expectations by Elmegreen (1993), observations by Wong \& Blitz (2002) and Blitz \& Rosolowsky (2006), and simulations by Robertson \& Kravtsov (2008). In its simplest form, this is a variation on the classical Schmidt law, i.e., $\mathrm{R}_{m o l}$ is set by gas volume density.

6) Power law fits of $\mathrm{R}_{m o l}$ to $\mathrm{P}_{h}$, radius, $\tau_{\text {orb }}$, and $\Sigma_{*}$ reproduce the observed star formation efficiency reasonably well in spiral galaxies but yield large scatter or higher than expected star formation efficiencies in the outer parts of dwarf galaxies, offering indirect evidence that differences between the dwarf and normal spiral subsample such as metallicity (dust), radiation field, and strong spiral shocks play a role in setting these relations.

\section{References}

Bigiel, F., Leroy, A., Walter, F., Brinks, E., de Blok, W. J. G., Madore, B., \& Thornley, M. D. 2008, arXiv:0810.2541

Blitz, L. \& Rosolowsky, E. 2004, ApJL, 612, L29

Blitz, L. \& Rosolowsky, E. 2006, ApJ, 650, 933

de Blok, W. J. G. \& Walter, F. 2006, AJ, 131, 363

Elmegreen, B. G. 1993, ApJ, 411, 170

Elmegreen, B. G. 1997, Revista Mexicana de Astronomia y Astrofisica Conference Series, 6, 165 Gil de Paz, A., et al. 2007, ApJS, 173, 185

Helfer, T. T., Thornley, M. D., Regan, M. W., Wong, T., Sheth, K., Vogel, S. N., Blitz, L., \& Bock, D. C.-J. 2003, ApJS, 145, 259

Hunter, D. A., Elmegreen, B. G., \& Baker, A. L. 1998, ApJ, 493, 595

Kennicutt, R. C., Jr. 1998, ARAA, 36, 189

Kennicutt, R. C., Jr. 1998, ApJ, 498, 541

Kennicutt, R. C., Jr., et al. 2003, PASP, 115, 928

Krumholz, M. R. \& McKee, C. F. 2005, ApJ, 630, 250

Leroy, A. K., Walter, F., Brinks, E., Bigiel, F., de Blok, W. J. G., Madore, B., \& Thornley, M. D. 2008, arXiv:0810.2556

Leroy et al. 2009, AJ submitted

Martin, C. L. \& Kennicutt, R. C., Jr. 2001, ApJ, 555, 301

Robertson, B. E. \& Kravtsov, A. V. 2008, ApJ, 680, 1083 
Schaye, J. 2004, ApJ, 609, 667

Skillman, E. D. 1987, NASA Conference Publication, 2466, 263

Walter, F., Brinks, E., de Blok, W. J. G., Bigiel, F., Kennicutt, R. C., Jr., Thornley, M. D., \& Leroy, A. K. 2008, arXiv:0810.2125

Wong, T. \& Blitz, L. 2002, ApJ, 569, 157

Wyse, R. F. G. 1986, ApJL, 311, L41

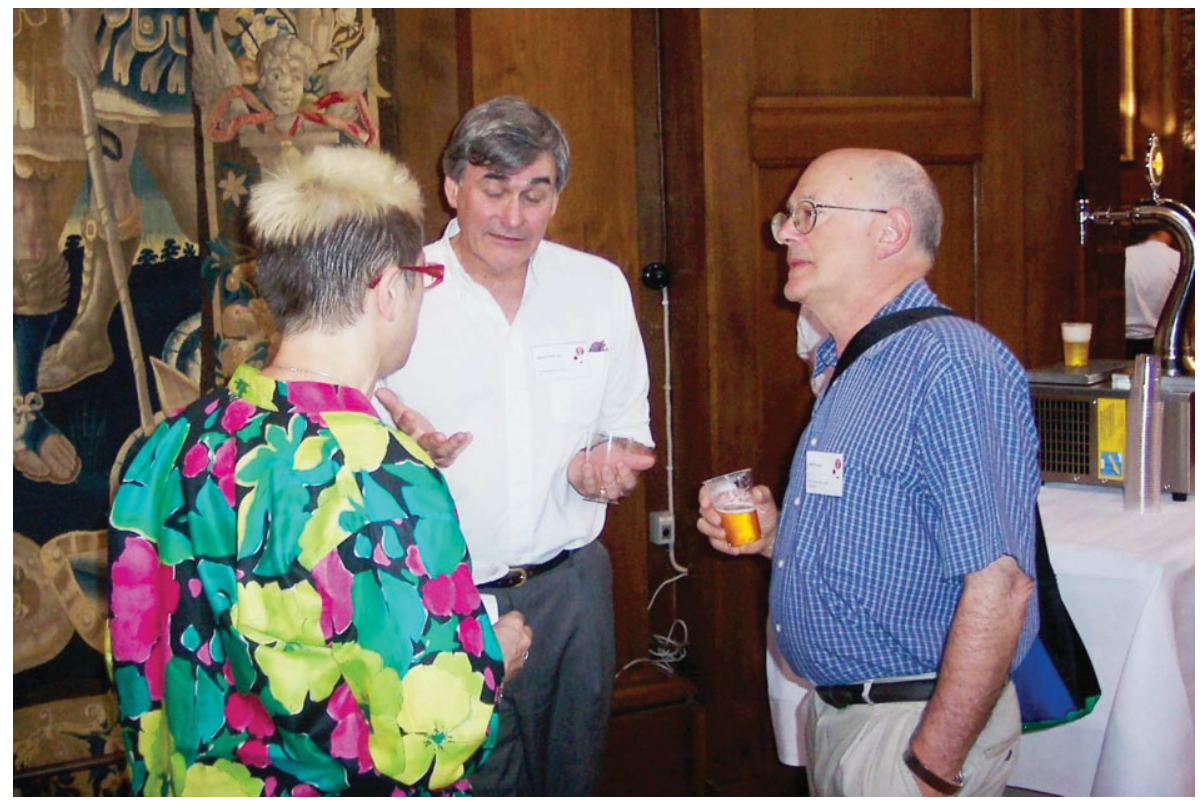

Linda Sparke, Gerry Gilmore and Ken Freeman discussing at the welcome reception. 